\title{
Microbial diversity and methanogenic potential in a high temperature natural gas field in Japan
}

\author{
Hanako Mochimaru $\cdot$ Hideyoshi Yoshioka $\cdot$ Hideyuki Tamaki · \\ Kohei Nakamura · Nobuyuki Kaneko · Susumu Sakata · Hiroyuki Imachi · \\ Yuji Sekiguchi $\cdot$ Hiroo Uchiyama $\cdot$ Yoichi Kamagata
}

Published online: 24 May 2008

(C) Springer 2008

\section{Erratum to: Extremophiles (2007) 11:453-461 \\ DOI 10.1007/s00792-006-0056-8}

The unit of acetate concentration in Table 1 and the reference in the text was shown incorrectly. The unit should be mmol $1^{-1}$. This does not affect any of our conclusions.

The online version of the original article can be found under doi:10.1007/s00792-006-0056-8.

H. Mochimaru $\cdot$ H. Uchiyama

Graduate School of Life and Environmental Sciences,

University of Tsukuba, Tsukuba,

Ibaraki 305-8572, Japan

H. Mochimaru $\cdot$ H. Tamaki $\cdot$ K. Nakamura

Y. Sekiguchi · Y. Kamagata $(\bowtie)$

Institute for Biological Resources and Functions,

National Institute of Advanced Industrial Science and Technology (AIST), 1-1-1 Higashi, Tsukuba,

Ibaraki 305-8566, Japan

e-mail: y.kamagata@aist.go.jp

\section{Y. Kamagata}

Research Institute of Genome-Based Biofactory,

National Institute of Advanced Industrial Science

and Technology (AIST), Sapporo, Hokkaido 062-8517, Japan

H. Yoshioka · N. Kaneko · S. Sakata

Institute for Geo-resources and Environment,

National Institute of Advanced Industrial Science

and Technology (AIST), Tsukuba, Ibaraki 305-8566, Japan

H. Imachi

Subground Animalcule Retrieval (SUGAR) Program,

Extremobiosphere Research Center,

Japan Agency for Marine-Earth Science \& Technology

(JAMSTEC), Yokosuka, Kanagawa 237-0061, Japan 